\title{
A Trend Analysis of Case Reports and Case Series on Hwa-byung in Korea
}

\author{
Soo-Hyun Sung ${ }^{1}$, Jong-Hyun Park ${ }^{2}$, Young-Joon Lee ${ }^{3}$, Soo-Jin Park ${ }^{4}$, Eun-Jung Park ${ }^{5}$, \\ Chang-Hyun $\operatorname{Han}^{1}$ \\ ${ }^{1}$ Department of Clinical Research Division, Korea Institute of Oriental Medicine \\ ${ }^{2}$ Department of Pathology, College of Korean Medicine, Daegu Haany University \\ ${ }^{3}$ Department of Preventive Medicine, College of Korean Medicine, Daegu Haany University \\ ${ }^{4}$ Department of Anatomy and Histology, College of Korean Medicine, Daegu Haany University \\ ${ }^{5}$ Information System Unit, Samsung Securities
}

\begin{abstract}
Objectives: This study aimed to provide a basis for treatment of Hwa-byung, a mental disorder frequently found among Koreans, by analysing published case reports and case series.

Methods: In June 2015, a literature search for case reports and case series describing interventions for Hwa-byung was conducted in 7 databases for research publications in Korea, using Korean and English terms.

Results: Thirty-two papers, reporting 439 cases of Hwa-byung, met the inclusion criteria. Since the first case report on Hwa-byung in 1996, case reports were continuously published until 2012. A total of 22 interventions were described, of which herbal medicine $(71.9 \%)$, acupuncture $(65.6 \%)$, and moxibustion $(34.4 \%)$ were the most frequently described interventions. Twenty-five papers reported patterns, for which liver qi depression (28\%) was most frequently used. A total of 48 outcome measures were used in 32 papers, of which Beck Depression Index (46.9\%) and Symptom change $(43.8 \%)$ were used in at least 10 papers.

Conclusions: Since only 32 case reports/case series have been published on Hwa-byung in Korea, a strong basis for recommending standardized treatments is lacking. Therefore, a sufficient number of clinical studies, in particular randomized clinical trials (RCTs), are warranted to provide a clinical basis for treatment of Hwa-byung.
\end{abstract}

$\overline{\text { Key Words }}$ : hwa-byung, anger syndrome, korean medicine

\section{Introduction}

According to the "Diagnostic and Statistical Manual of Mental Disorders-IV" published by the American Psychiatric Association, Hwa-byung is an anger syndrome- a Korean folk syndrome, caused by suppression of anger ${ }^{1)}$. In addition, Hwa-byung has been mentioned as a disease in oriental medicine in the "Korean Standard Classification of Diseases"2). Taken together, these facts indicate that Hwa-byung has been recognized as a disease found commonly in Koreans.

Although Hwa-byung is usually explained in relation to Korean culture that is suppressive and discourages one from expressing oneself, it is not limited to Koreans. As a result of various studies investigating this disease, Hwa-byung is currently considered a universal disease caused by uncontrollable anger or long-term suppression of anger ${ }^{3)}$. Hwa-byung is also called Wool-hwabyung, in which the anger

\footnotetext{
- Received : 30 November $2015 \quad \cdot$ Revised : 15 December 2015 - Accepted : 21 December 2015

- Correspondence to : Chang-Hyun Han

1672, Yuseongdae-ro, Yuseong-gu, Daejeon, 34054, Korea

Tel : +82-42-868-9638, Fax : +82-42-868-9463, E-mail : chhan@kiom.re.kr
} 
pent-up inside an individual is expressed as wrath. Hwa-byung patients experience symptoms including oppression in the chest, upper body heat, anxiety, palpitations, depression, and flush face; long-term patients tend to be more prone to depression ${ }^{4}$.

The prevalence of Hwa-byung was $4.1 \%$ in Korea, and it was more prevalent among middle-aged people and women ${ }^{5-7)}$. In addition, a study reported that Hwa-byung was a disease distinct from psychiatric disorders such as depression and anxiety, and 15\% of patients with Hwa-byung were not classified under other psychiatric disorders8?.

Different kinds of studies have been carried out in Korea to study Hwa-byung, e.g. literature reviews to investigate its concept ${ }^{9-10)}$, clinical studies to investigate characteristics of patients with Hwa-byung ${ }^{11-12}$, studies for pattern standardization ${ }^{13-14)}$, and studies on development of standard outcome measures ${ }^{15-16)}$. In contrast, there have been few studies outside Korea, and most of the papers published in English were reviews by Korean doctors or Korean medicine doctors to introduce the concept of Hwa-byung ${ }^{17)}$, clinical studies conducted on patients with Hwa-byung ${ }^{18)}$, and comparative studies between Hwa-byung and other diseases ${ }^{19}$.

Therefore, the aim of the present study was to investigate the current situation of case reports and case series among clinical studies papers on Hwa-byung published in Korea, and analyse outcome measures, interventions, and patterns for Hwa-byung. This would eventually aid in preparing a basis for standardization of diagnosis and treatment of Hwa-byung.

\section{Methods}

\section{Data sources}

In order to investigate Korean papers on Hwa-byung, we selected the following 7 databases in accordance with 'NECA's guidance for undertaking systematic reviews and meta-analyses for intervention"20): Research Information Service System (RISS), Korean Medical Database, Korea Citation Index (KCI), Korea Med, National Discovery for Science Leaders (NDSL), National Assembly Library and DBpia. The following search terms were used: 'Wool-hwabyung (울화병)', 'Hwabyung(화병)', 'Hwa byung', 'Anger Syndrome' and 'Anger Disorder', both in Korean and English. The data for analyses were extracted in June 2015, with no limits on search period.

\section{Study selection}

We selected case reports and case series describing interventions among papers on clinical studies of Hwa-byung. News items, letters, randomized clinical trials (RCTs), controlled clinical trials (CCTs), qualitative studies, reviews, surveys, and interviews were excluded. In addition, case reports and case series not discussing interventions were also excluded (Fig. 1).

\section{Data extraction}

Two authors (Chang-Hyun Han and Soo-Hyun Sung) selected papers meeting the inclusion criteria, which were then classified into categories. The two authors independently extracted and integrated information about authors, year of publication, age, gender, number of patients, intervention, outcome measure, conclusion, and pattern. Disagreements between the two authors were resolved by discussion until a consensus was reached.

\section{Results}

\section{Search Process and Result}

As a result of the initial searches, 14241 papers were found, among which duplicate publications, papers not conforming to a basic paper format (without abstracts or references), and papers not related to Hwa-byung were excluded, resulting in a 


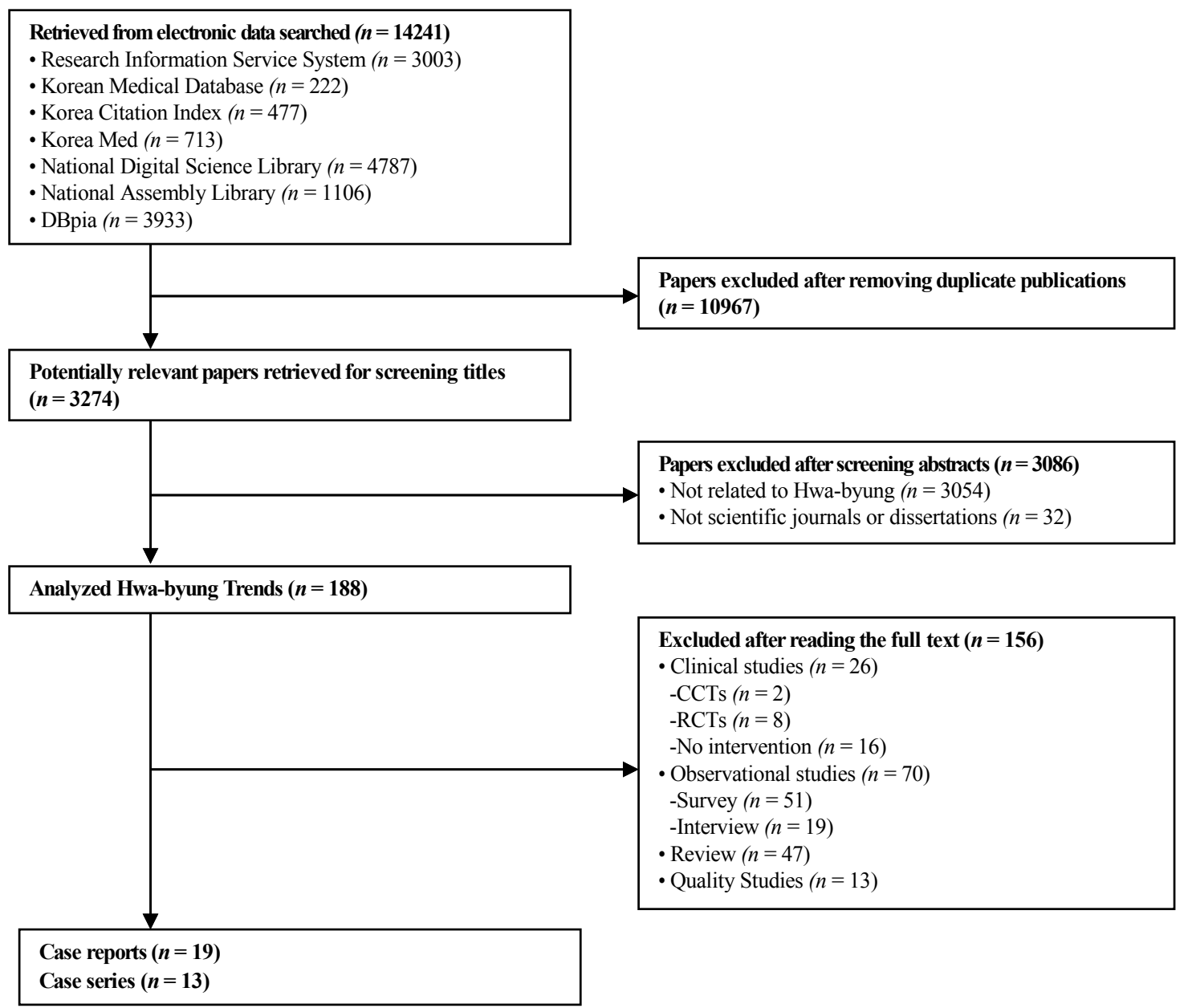

Fig. 1. Flowchart of the study selection process

CCTs: Controlled Clinical Trials; RCTs: Randomized Clinical Trials.

total of 188 papers. Of them, a total of 32 papers (19 case reports and 13 case series), describing 439 cases of Hwa-byung, met the inclusion criteria. The characteristics of the selected case reports and case series are presented in Tables 1 and 2. Names of acupuncture points were written based on the "Standard Acupuncture Nomenclature", names of prescriptions were based on the "Compilation of Formulas and Medicinal Addendum", and names of patterns and symptoms were based on the "WHO International Standard Terminologies on Traditional
Medicine in the Western Pacific Region" and the "Standard Terminologies on Korean Medicine".

\section{Number of papers and cases by year.}

The first case report was published in 1996; there was no case report or case series published after 2013. Four case reports and 4 case series the highest numbers - were published in 2005 and 2012, respectively. The maximum number of cases reported in a year was 147 in 2006 (Fig. 2). 


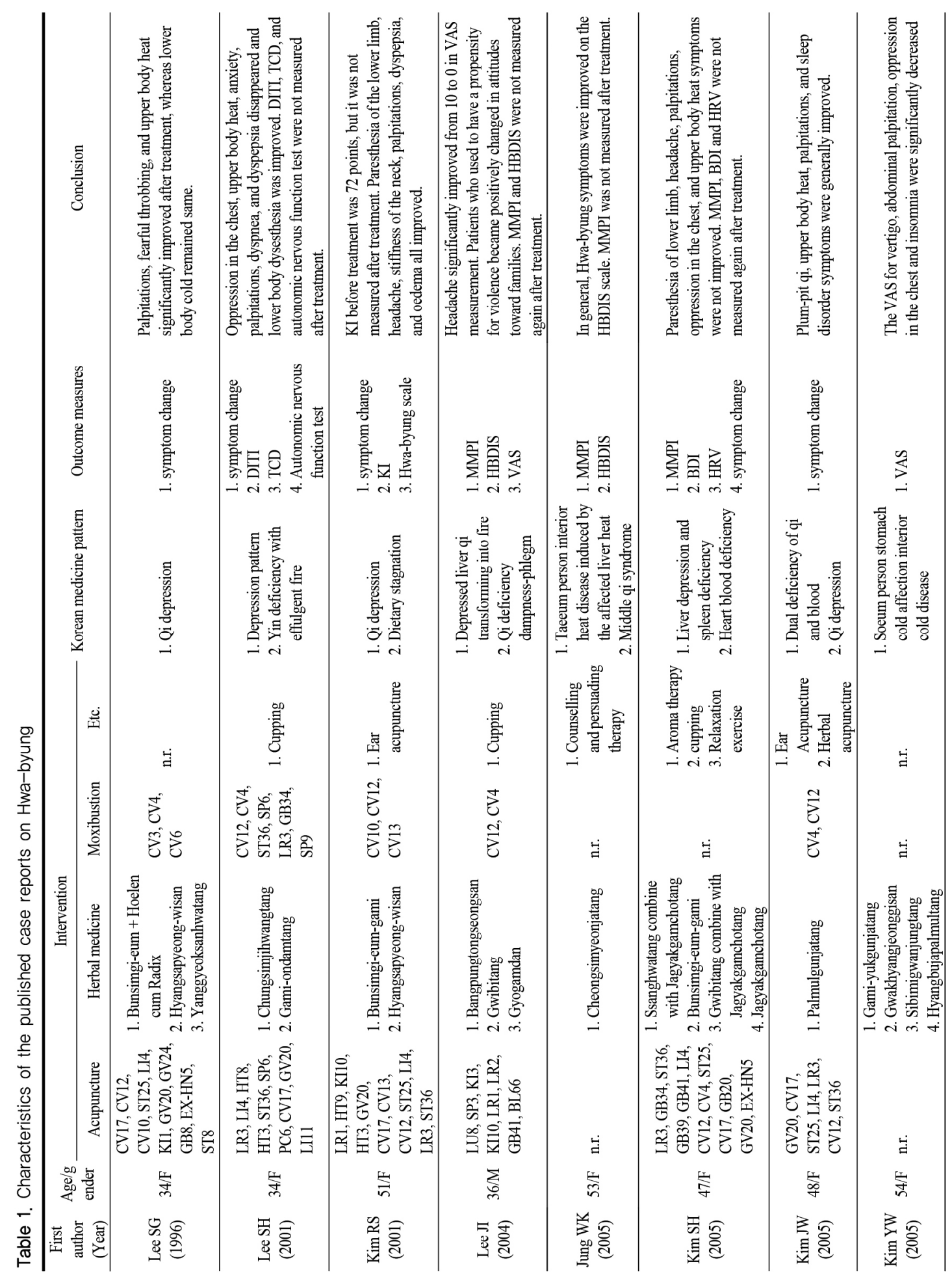




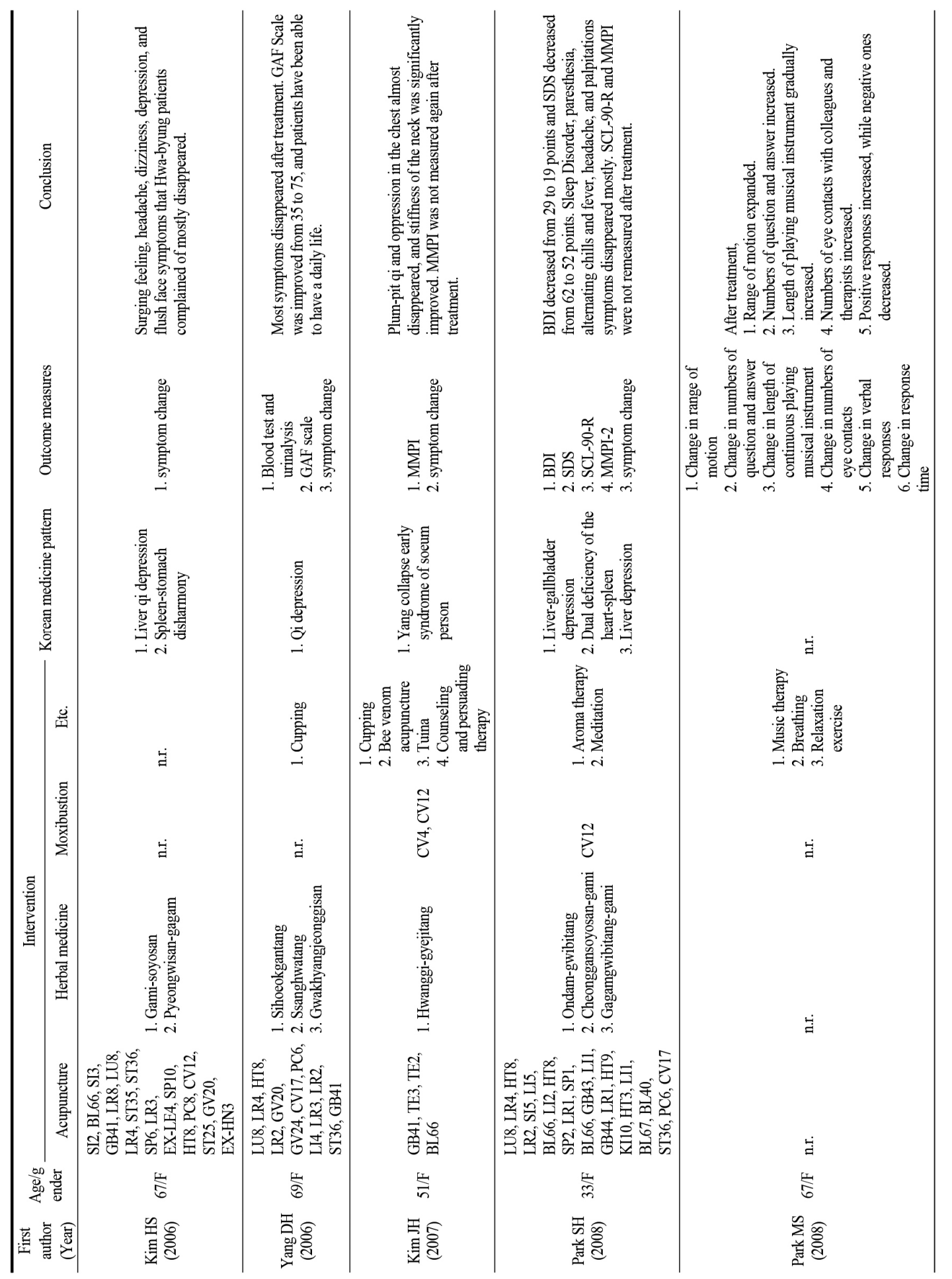




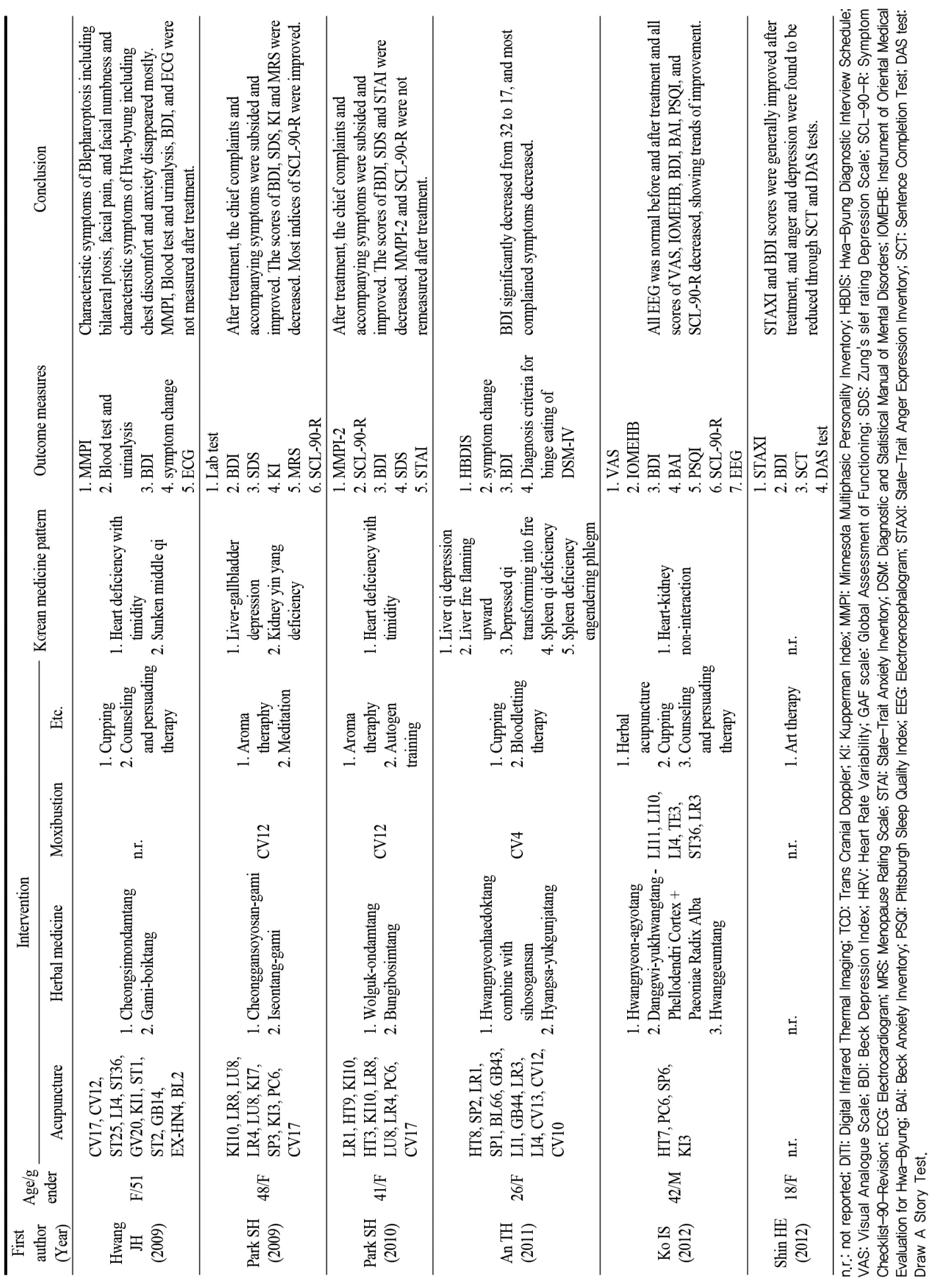




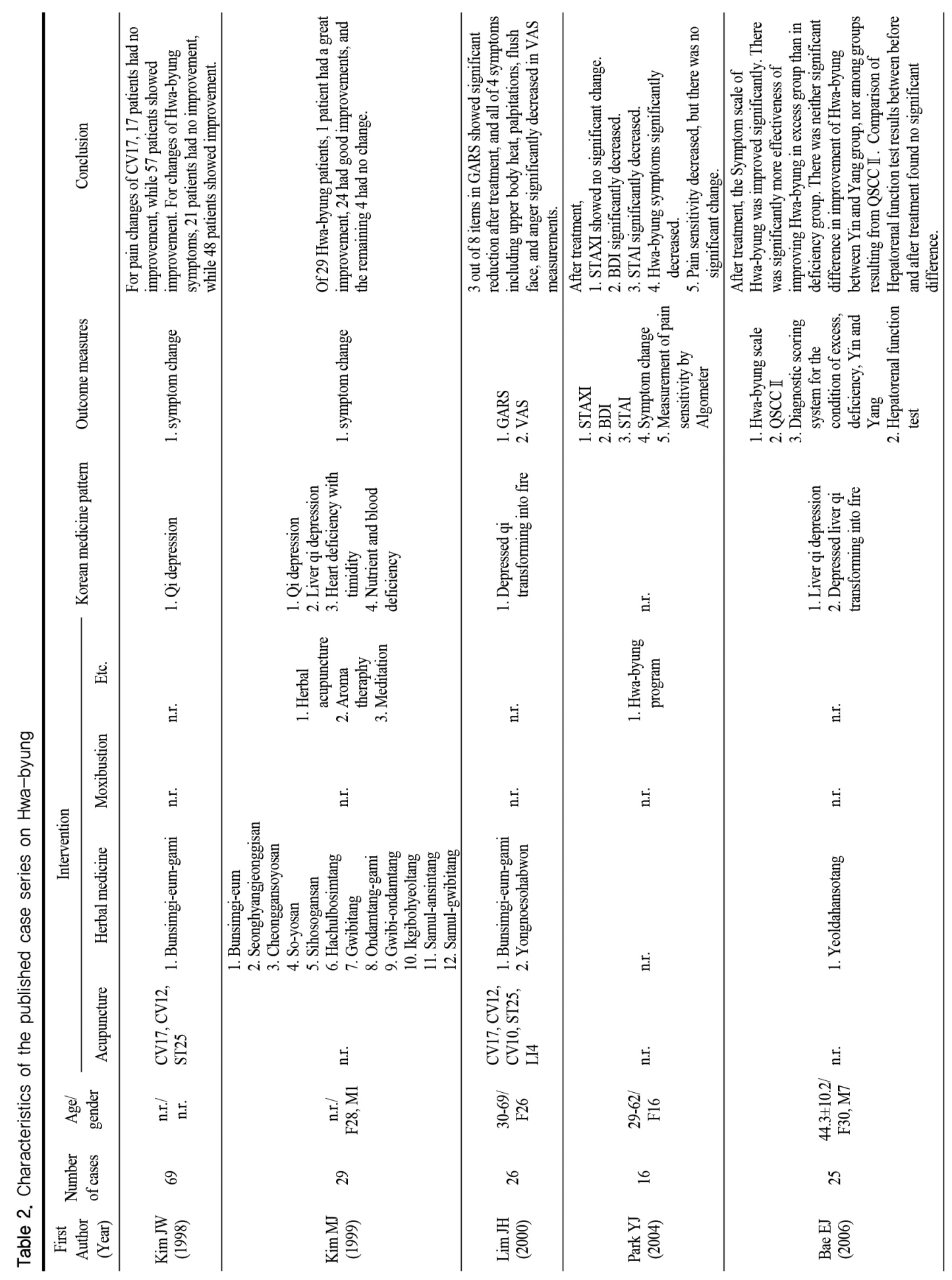




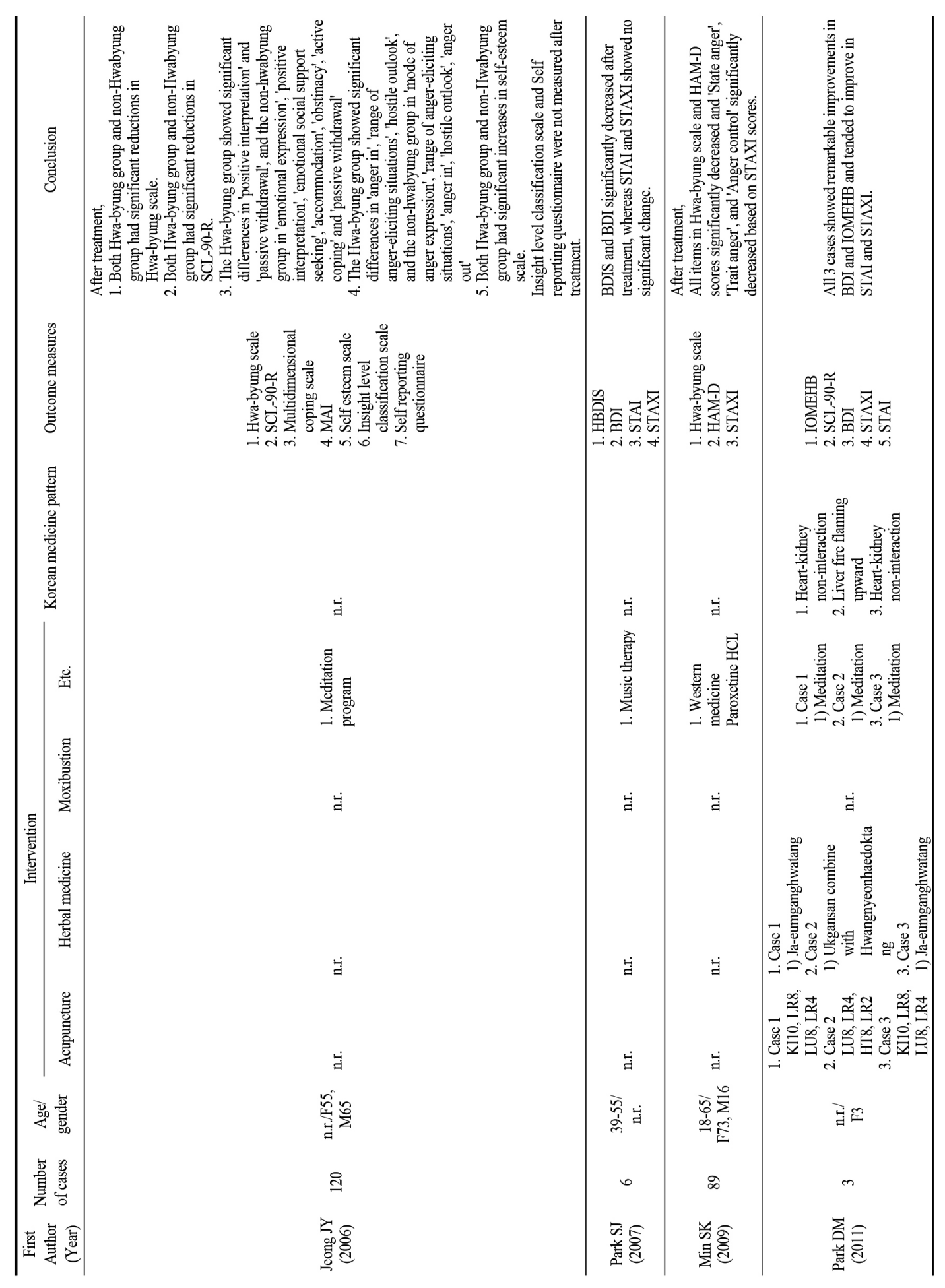




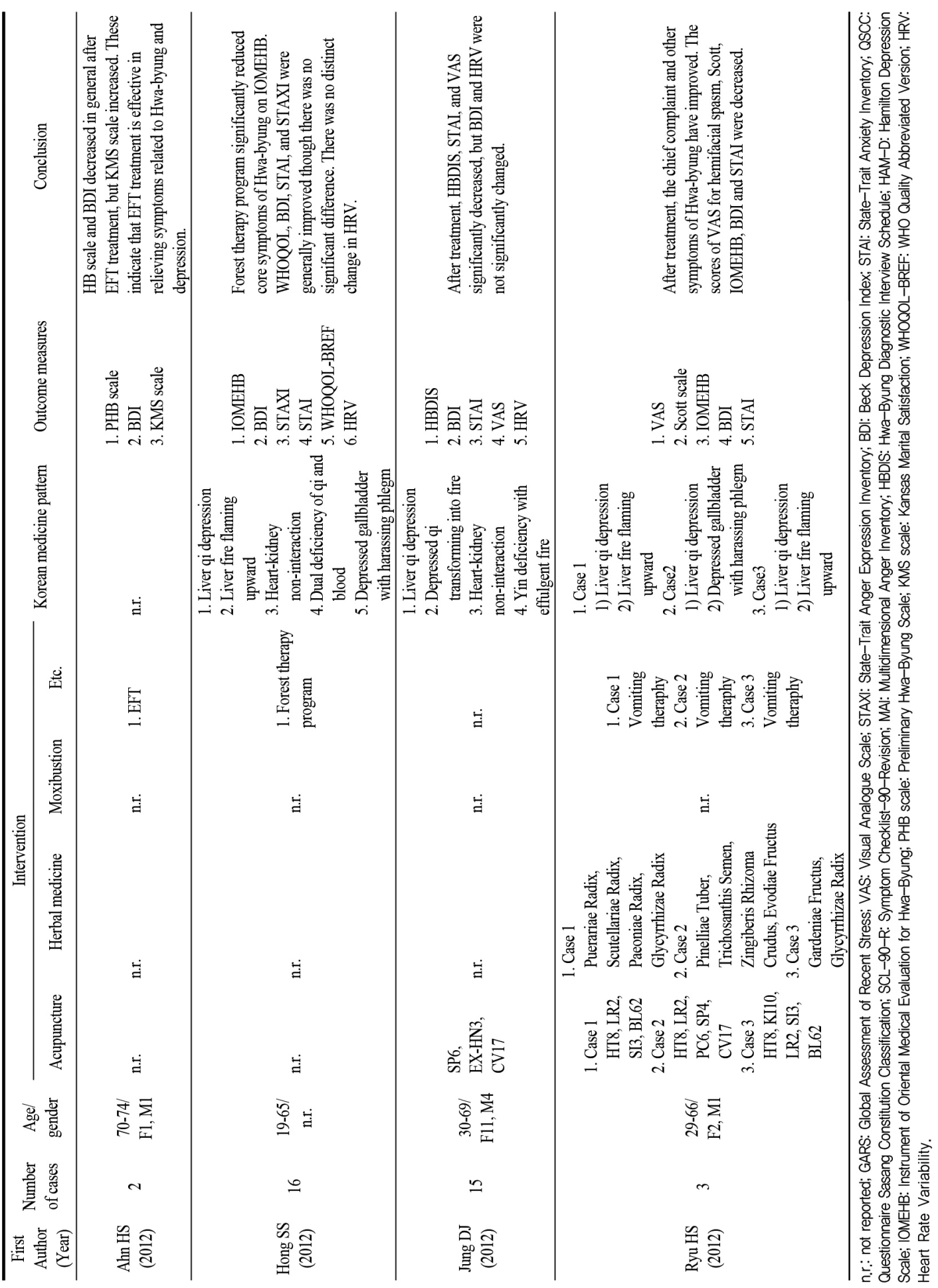




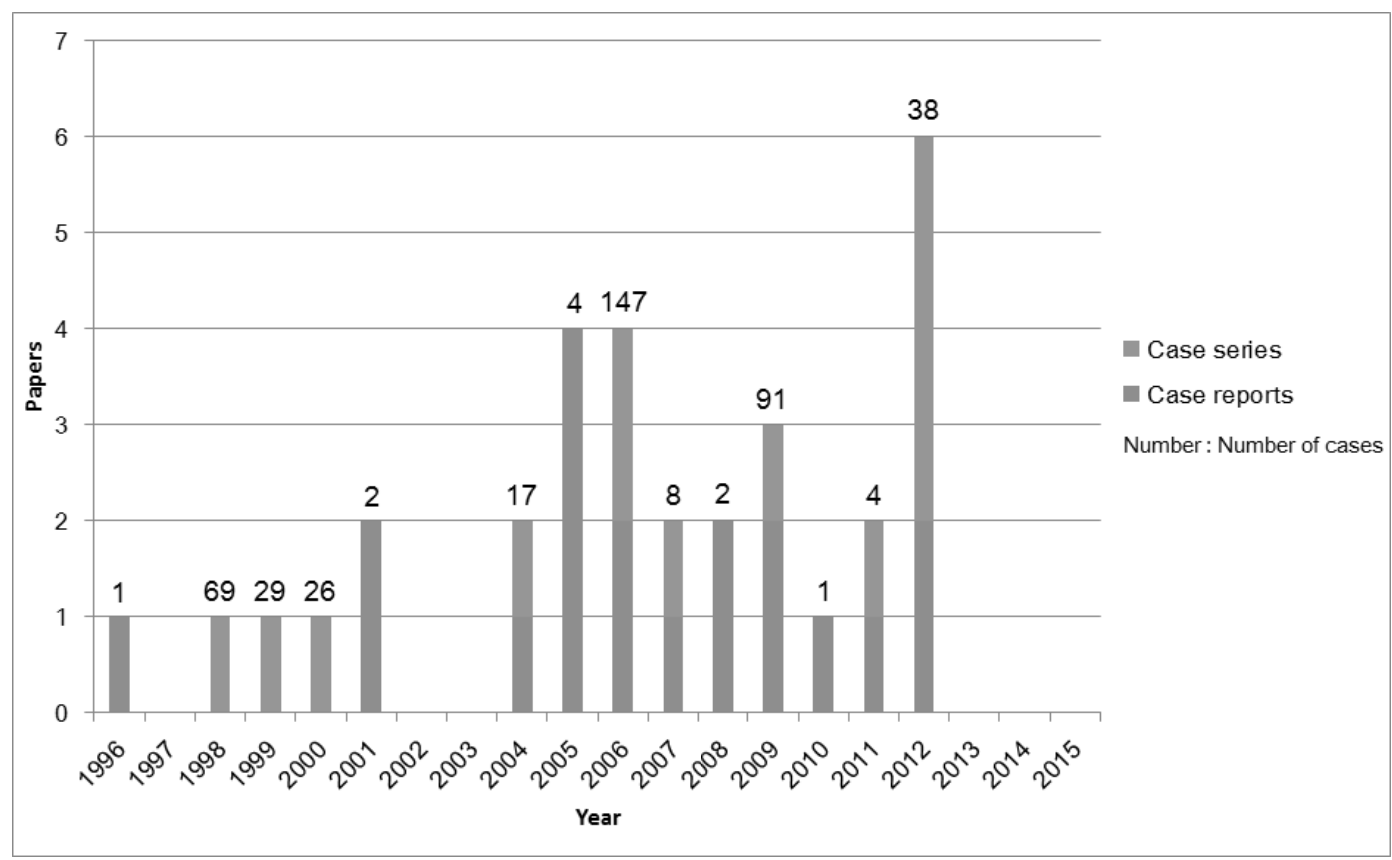

Fig. 2. Trend of papers and cases of Hwa-byung by year

\section{Interventions.}

A total of 22 interventions were described in the 32 papers, of which herbal medicine, acupuncture, and moxibustion were described in 23, 21, and 11 papers, respectively. Interventions described in at least 2 papers are presented in Table 3.

Table 3. Interventions reported in case reports and case series on Hwa-byung.

\begin{tabular}{c|c}
\hline Intervention & Number of papers \\
\hline Herbal medicine & $23(71.9 \%)$ \\
Acupuncture & $21(65.6 \%)$ \\
Moxibustion & $11(34.4 \%)$ \\
Cupping & $8(25 \%)$ \\
Aroma therapy & $5(15.6 \%)$ \\
Meditation & $5(15.6 \%)$ \\
Counselling and persuading therapy & $4(12.5 \%)$ \\
Herbal acupuncture & $3(9.4 \%)$ \\
Ear acupuncture & $2(6.3 \%)$ \\
Relaxation exercise & $2(6.3 \%)$ \\
Music therapy & $2(6.3 \%)$ \\
\hline
\end{tabular}

1) Herbal Medicines

A total of 51 herbal medicines were mentioned in 23 papers, of which Bunsimgi-eum and Cheonggansoyosan appeared in 6 and 3 papers, respectively, and each of Gwakhyangjeonggisan, Hyangsapyeong-wisan, and Gwibitang appeared in 2 papers (Table 4).

Table 4. Herbal medicines mentioned in case reports and case series on Hwa-byung

\begin{tabular}{c|c|r}
\hline Herbal Medicine & Korean name & $\begin{array}{c}\text { Number of } \\
\text { papers }\end{array}$ \\
\hline Bunsimgi-eum & 分心氣飲 & $6(26.1 \%)$ \\
Cheonggansoyosan & 淸肝逍遙散 & $3(13 \%)$ \\
Gwakhyangjeonggisan & 嚾香正氣散 & $2(8.7 \%)$ \\
Hyangsapyeong-wisan & 香砂本胃散 & $2(8.7 \%)$ \\
Gwibitang & 歸脾湯 & $2(8.7 \%)$ \\
\hline
\end{tabular}

2) Korean Medicine pattern

Of the 32 papers, there were 3, 22, and 7 papers with visceral patterns, four constitutional patterns, and without pattern, respectively. A total of 28 
Korean medicine patterns were described in 25 papers, of which liver qi depression and qi depression were described in 7 and 6 papers, respectively, and each of heart-kidney non-interaction and liver fire flaming upward was described in 4 papers. Korean medicine patterns described in at least 2 papers are presented in Table 5.

Table 5. Korean Medicine patterns described in case reports and case series on Hwa-byung

\begin{tabular}{|c|c|c|}
\hline Korean Medicine pattern & $\begin{array}{c}\text { Korean } \\
\text { name }\end{array}$ & $\begin{array}{c}\text { Number of } \\
\text { papers }\end{array}$ \\
\hline Liver qi depression & 肝氣鬱結 & $7(28 \%)$ \\
\hline Qi depression & 氣鬱 & $6(24 \%)$ \\
\hline Heart-kidney non-interaction & 心腎不交 & $4(16 \%)$ \\
\hline Liver fire flaming upward & 肝火上炎 & $4(16 \%)$ \\
\hline Heart deficiency with timidity & 心膽虛怯 & $3(12 \%)$ \\
\hline Depressed qi transforming into fire & 氣鬱化火 & $3(12 \%)$ \\
\hline
\end{tabular}

\section{3) Acupuncture points}

A total of 62 acupuncture points were mentioned in 21 papers, of which CV17 appeared in 14 papers and each of KI10, LI4, and CV12 appeared in 9 papers. Acupuncture points mentioned in at least 6 papers are presented in Table 6. Among the case

Table 6. Acupuncture points mentioned in case reports and case series on Hwa-byung

\begin{tabular}{c|c|c}
\hline $\begin{array}{c}\text { Acupuncture } \\
\text { point }\end{array}$ & Korean name & Number of papers \\
\hline CV17 & 膻中 & $14(66.7 \%)$ \\
KI10 & 陰谷 & $9(42.9 \%)$ \\
LI4 & 合谷 & $9(42.9 \%)$ \\
CV12 & 中脘 & $9(42.9 \%)$ \\
GV20 & 百會 & $8(38.1 \%)$ \\
ST36 & 足三里 & $8(38.1 \%)$ \\
ST25 & 天樞 & $8(38.1 \%)$ \\
LU8 & 經渠 & $7(33.3 \%)$ \\
PC6 & 內關 & $7(33.3 \%)$ \\
HT8 & 少府 & $7(33.3 \%)$ \\
LR3 & 太衝 & $7(33.3 \%)$ \\
LR4 & 中封 & $6(28.6 \%)$ \\
\hline
\end{tabular}

series, the one by Kim and Lee ${ }^{21)}$ reported 29 cases of Hwa-byung, but did not mention any acupuncture points.

4) Moxibustion points

A total of 15 moxibustion points were mentioned in 11 papers, of which CV12 and CV4 were mentioned in 8 and 6 papers, respectively, and each of ST36 and LR3 was mentioned in 2 papers (Table 7). Thirteen case series did not mention moxibustion.

Table 7. Moxibustion points mentioned in case reports and case series on Hwa-byung

\begin{tabular}{c|c|c}
\hline $\begin{array}{c}\text { Moxibustion } \\
\text { point }\end{array}$ & Korean name & Number of papers \\
\hline CV12 & 中脘 & $8(72.7 \%)$ \\
CV4 & 關元 & $6(54.5 \%)$ \\
ST36 & 足三里 & $2(18.2 \%)$ \\
LR3 & 太衝 & $2(18.2 \%)$ \\
\hline
\end{tabular}

\section{Outcome measures}

A total of 48 outcome measures were reported, including Beck Depression Index (BDI) in 15 papers, symptom change in 14 papers, and Minnesota Multiphasic Personality Inventory (MMPI) and State-Trait Anxiety Inventory (STAI) in 7 papers each. The outcome measures reported in at least 3 papers are presented in Table 8. Hwa-byung outcome measures made in Korea include the Hwa-byung Diagnostic Interview Schedule (HBDIS), Hwa-byung scale, Instrument of Oriental Medical Evaluation for Hwa-byung (IOMEHB), and Preliminary Hwa-byung Scale (PHB scale), of which the Hwa-byung scale is not a structured instrument, but an instrument made through independent development or modification of the HBDIS or IOMEHB. HBDIS is not a general outcome measure for measuring the severity of symptoms but a structured questionnaire tool for diagnose Hwa-byung. 
Table 8. Outcome measure reported in case reports and case series on Hwa-byung

\begin{tabular}{c|c}
\hline Outcome measure & Number of papers \\
\hline BDI & $15(46.9 \%)$ \\
symptom change & $14(43.8 \%)$ \\
MMPI & $7(21.9 \%)$ \\
STAI & $7(21.9 \%)$ \\
SCL-90-R & $6(18.8 \%)$ \\
VAS & $6(18.8 \%)$ \\
STAXI & $6(18.8 \%)$ \\
HBDIS & $5(15.6 \%)$ \\
Hwa-byung scale & $5(15.6 \%)$ \\
IOMEHB & $4(12.5 \%)$ \\
SDS & $3(9.4 \%)$ \\
HRV & $3(9.4 \%)$ \\
\hline
\end{tabular}

BDI: Beck Depression Index; MMPI: Minnesota Multiphasic Personality Inventory; STAl: State-Trait Anxiety Inventory; SCL-90-R: Symptom Checklist-90-Revision; VAS: Visual Analogue Scale; STAXI: State-Trait Anger Expression Inventory; HBDIS: Hwa-Byung Diagnostic Interview Schedule; IOMEHB: Instrument of Oriental Medical Evaluation for Hwa-Byung; SDS: Zung's Self-rating Depression Scale; HRV: Heart Rate Variability.

\section{Discussion}

Hwa-byung was first mentioned as a disease concept in “Gyungakjeonseo (景岳全書)” in 162422), and the first study on Hwa-byung in Korea was a literature review by Lee ${ }^{23)} 1977$ that explained the concept of Hwa-byung. The first clinical studies of Hwa-byung was a case report by $\mathrm{Lee}^{24}$ 1996, in which Hwa-byung was diagnosed and evaluated on the basis of symptom change in patients. Although the "Clinical evidence of Korean medicine", published by the Association of Korean Medicine and the Korea Institute of Oriental Medicine in 2014, proposed a study design for studies on Hwa-byung, interventions, outcome measures, and results, since initially found 373 papers classified and analysed data, a complete enumeration survey was not conducted $^{25)}$. The clinical practice guidelines for Hwa-byung, published in 2013 in Korea, were established based on existing papers on Hwa-byung and the advice of experts. However, these were just a summary of papers corresponding to each content and recommendation based on expert advice, so it was difficult to consider these as standard guidelines for the treatment of Hwa-byung ${ }^{26)}$. Hence, in this study, we investigated all case reports and case series on Hwa-byung published in Korea, in order to analyse and report outcome measures, various interventions, and frequencies of patterns.

The first case report on Hwa-byung in Korea was published in 1996. Since then, papers on Hwa-byung have been published continuously until 2012. The "Anger syndrome" related to the Korean culture was introduced in the "Diagnostic and Statistical Manual of Mental Disorders-IV," corresponding to the international standard in $1995^{1)}$. Since then, several case reports and case series describing standard diagnosis, evaluation, and treatment of Hwa-byung have been published in Korea. No case reports or case series have been published after 2013 when the clinical practice guidelines for Hwa-byung were published in Korea. This is because after the publication of these guidelines, RCTs were conducted and published, rather than case reports and case series ${ }^{27-9)}$. In addition, it has become difficult to publish the case report and case series on Journal of Oriental Neuropsychiatry, which previously accepted a lot of papers of Hwa-byung because of its renewal internal guidelines and many reports of Hwa-byung described similar patients' symptoms.

A total of 32 papers reported 439 cases of Hwa-byung. These numbers are still too small to provide a strong basis for standardization of Hwa-byung treatment. Therefore, standardized guidelines for Hwa-byung should be developed through clinical studies based on the clinical practice guidelines.

Of the 32 papers, herbal medicine, acupuncture, moxibustion, and cupping were described in 23 (71.9\%), 21 (65.6\%), 11 (34.4\%), and 8 papers (25\%), respectively. This seems to be consistent with 
opinions of experts from a survey, in that patients with Hwa-byung were treated mainly with acupuncture and herbal medicine, and moxibustion, cupping, and psychotherapy were used as complementary treatments $^{30)}$.

Since occurrence of Hwa-byung is significantly affected by suppression of anger without control $^{31)}$, it is highly important to treat both physical and mental symptoms. Of the interventions used in the papers, treatments including meditation, relaxation exercises, and breathing belong to the mind body medicine defined by the National Center for Complementary and Alternative Medicine (NCCAM) and are considered appropriate for Hwa-byung treatment ${ }^{32}$.

Hwa-byung is highly correlated with relationships and has a high recurrence rate after treatment ${ }^{33)}$. This may be because the relationship of the patient with the people who provoked the uncontrollable anger were not dealt with, even though the symptoms were treated. Hence, if a patient is treated together with the person whom he/she regards as the cause, the treatment effect would last longer.

The most frequent pattern was liver qi depression, mentioned in 7 papers(28\%), followed by qi depression in 6 papers(24\%), each of heart-kidney non-interaction and liver fire flaming upward in 4 papers (16\%), and each of heart deficiency with timidity and depressed qi transforming into fire in 3 papers $(12 \%)$.

A total of 51 herbal medicines were mentioned in 23 papers, which include Bunsimgi-eum in 6 papers (26.1\%), Cheonggansoyosan in 3 papers (13\%), and Gwakhyangjeonggisan, Hyangsapyeong-wisan and Gwibitang in 2 papers (8.7\%) each.

For correlations of pattern with prescription, Gwakhyangjeonggisan was used for qi depression, Bunsimgi-eum for liver qi depression, qi depression, heart-kidney non-interaction, and depressed qi transforming into fire, Cheonggansoyosan for liver fire flaming upward and depressed qi transforming into fire, Gwibitang for heart deficiency with timidity, and Hyangsapyeong-wisan for digestive symptoms by qi depression and liver qi depression.

Through literature reviews and expert consultations, Yim $^{14)}$ presented 5 major patterns - liver qi depression, liver fire flaming upward, heart-kidney non-interaction, dual deficiency of qi and blood, and depressed gallbladder with harassing phlegm - and their symptoms in a table, and the "Clinical Guidelines for Hwa-byung" reported that the reliability of the 5 pattern instruments was secured based on clinical studies with 159 patients $^{26}$. Since patterns play an important role in the selection of treatment methods more confidently, if more pattern data are accumulated through more clinical studies, it will be possible to systematize Hwa-byung treatment methods by pattern.

Acupuncture points were mentioned in 21 papers - CV17 in 14 papers (66.7\%), KI10, LI4, and CV12 in each 9 papers (42.9\%), and GV20, ST36, and ST25 in each 8 papers (38.1\%). These results are in agreement with the acupuncture points for Hwa-byung treatment presented in "Neuropsychiatry of Korean Medicine," the textbook commonly used in the colleges of oriental medicine in Korea ${ }^{34)}$.

It is thought that since CV17 clears the lungs, resolves phlegm and moves qi to soothe the chest ${ }^{35)}$, CV17 was used for treatment of symptoms such as chest pain, oppression in the chest, and palpitations. Considering that $99 \%$ of patients with Hwa-byung experienced chest symptoms, CV17 was the most frequently used acupuncture point ${ }^{26)}$. Since KI10 tonifies the kidney and clears heat ${ }^{35)}$, it appears to be used for treatment of symptoms including upper body heat, headache, and hyperemia. LI4 clears and discharges lung qi and unblocks the meridian and activates collaterals ${ }^{36}$, so that it seemed to be used for treatments of symptoms like upper body heat, headache, chest discomfort, and anxiety. CV12 regulates middle energizer and upward and downward of middle $\mathrm{qi}^{\mathrm{i5})}$, so that it seems to be used for treatment of symptoms such as heart-kidney non-interaction or qi depression. 
Moxibustion points were mentioned in 11 papers: CV12 and CV4 were used in $8(72.7 \%)$ and 6 papers $(54.5 \%)$, respectively. The "Clinical guidelines for Hwa-byung IV. (Medications \& Acupuncture and Moxibustion)" stated that since Hwa-byung patients are likely to have cold abdomen due to movement of heat to the upper body, they need to be treated by circulation of qi blood through thermal stimulation of abdomen $^{37}$. Since both of CV12 and CV4 are abdominal acupuncture points, this indicates that moxibustion on these points was intended to improve the upper heat and lower cold condition through thermal stimulation of the abdomen.

Of the 48 outcome measures reported in the 32 papers, BDI was reported in 15 papers $(46.9 \%)$ - the highest number. BDI has been continuously used throughout the years, which indicates that it is an instrument to identify improvement of depressive symptoms in patients with Hwa-byung. In addition, HBDIS and IOMEHB, which are Hwa-byung scales made in Korea as Hwa-byung outcome measures, were used in $5(15.6 \%)$ and $4(12.5 \%)$ papers, respectively. Various outcome measures were used for a single case. There have been a number of attempts at establishing outcome measures for Hwa-byung in Korea, which is indicative of the attention this disease has received.

In addition, 14 papers $(43.8 \%)$ - the second highest number - mentioned symptom change as an outcome measure, which indicates that Hwa-byung was treated based on subjective indices of patients, instead of objective diagnosis instruments. It indicates neurological psychiatric disorders measured by objective diagnosis instruments, but the final decision is based on symptoms changes of patients and experts' judgment. Of the outcome measures reported, heart rate variability (HRV) and digital infrared thermal imaging (DITI) were reported in $3(9.4 \%)$ and 1 paper (3.1\%), respectively. Jung IC et al. proposed DITI and HRV as instruments to objectively measure Hwa-byung, other than psychological scales ${ }^{38)}$, but since the number of papers is insufficient, additional studies are necessary.

Currently, papers reporting clinical studies on Hwa-byung are still lacking, so the "Clinical Guidelines for Hwa-byung" published in Korea in 2013 do not have a strong basis. Therefore, it is necessary to perform more clinical studies that can be used as a basis for the establishment of clinical guidelines, in particular, RCTs, which have a higher reliability. This would enable researchers to verify, revise, and complement the clinical practice guidelines for a higher completion.

\section{Conclusions}

We found that most of the papers on Haw-byung that were published in Korea were using Korean medicine interventions, such as acupuncture, herbal medicine, and moxibustion. Moreover, most of the case reports and case series stated that Korean medicine interventions were effective in treating Hwa-byung. Therefore, focusing on Korean medicine interventions while constructing international standard treatment guidelines for Hwa-byung can be of great assistance in treating patients with Hwa-byung.

Conflicts of interest: The authors declare that there is no conflict of interest regarding the publication of this paper.

\section{Achknowledgement}

This study was supported by 'New Donguibogams' Compilation Project (K15500) of KIOM (Korea Institute of Oriental Medicine) in 2015.

\section{References}

1. American Psychiatric Association. Diagnostic and Satistical Manual of Mental Disorders 4th edition. Virginia: American Psychiatric Association. 
1995.

2. Statistics Korea. Korean Standard Classification of Diseases. Daejeon: Statistics Korea. Republic of Korea. 2010.

3. Kim WJ. Be Liberated from Hwa-byung. Seoul: Women News Publication. 2007.

4. Chon KK, Whang WW, Kim JW, Park HG. Emotional stress and Hwa-byung. Korean Journal of Health Psychology. 1997;2(1):168-185.

5. Lee JG, Lee JH. Study on the Prevalence of Hwa-byung Diagnosed by HBDIS in General Population in Kang-won Province. Journal of Oriental Neuropsychiatry. 2008;19(2):133-139.

6. Min SG, Kim JH. A Study on Hwa-byung in Bokil Island. Psychiatry Investigation. 1986;25(3): 459-466.

7. Kim HK, Park JY. Prevalence and Related Factors of Hwa-byung for the Aged Woman in Rural Community. Journal of Korea Community Health Nursing Academic Society. 2004;18(2): 234-242.

8. Park JH, Min SK, Lee MH. A Study on the Diagnosis of Hwa-byung. Journal of Korean Neuropsychiatric Association. 1997;36(3):496-502.

9. Min SK. A Study of the Concept of Hwa-byung. Journal of the Korean Neuropsychiatric Association 1989;28(4):604-616.

10. Park JS, Lyu YS. A Bibliographic Study about the Meaning of Hwa and Hwa-byung. Journal of Oriental Neuropsychiatry. 1997;8(1):183-200.

11. Ko CN, Lee KS. A Study about Apply DITI to Hwa-byung Patients. Journal of Oriental Medical Thermology. 2002;1(1):13-19.

12. Lee JG, Koh SB, Chang SJ, Park JK, Yu JS, Lee JH. A Comparative Study of HRV between Normal and Hwa-byung: Subject to Kang-won Province Inhabitants in 2006. Journal of Oriental Neuropsychiatry. 2008;19(3):171-178.

13. Lee HY, Kim JW, Park JH, Whang WW. A Study for Diagnosis and Pattern Identification of Hwa-byung. Journal of Oriental Neuropsychiatry.
2005;16(1):1-17.

14. Yim HJ, Kim SH, Lee SR, Jung IC. Study to Develop the Instrument of Pattern Identification for Hwa-byung. Journal of Oriental Physiology \& Pathology. 2008;22(5):1071-1077.

15. Kim JW. Development of Hwa-Byung Diagnostic Interview Schedule(HBDIS) and Its Validity Test. Korean Journal of Health Psychology. 2004;9(2):321-331.

16. Cheong MH, Lee SR, Kang WC, Jung IC. Preliminary Study to Develop the Instrument of Oriental Medical Evaluation for Hwa-byung. Journal of Oriental Neuropsychiatry. 2010;21(2): 141-155.

17. Suh S. Stories to be Told: Korean Doctors between Hwa-byung (Fire-illness) and Depression, 1970-2011. Culture, Medicine, and Psychiatry. 2013;37(1):81-104.

19. Kim SH, Park YC, Hong KE, Kang W, Lee SR, Jung IC. The Effect of Bunsimgi-eum on Hwa-byung: Randomized, Double Blind. Placebo Controlled Trial. Journal of Ethnopharmacology. 2012;144(2):402-407.

10. Lee J, Min SK, Kim KH. Kim B, Cho SJ, Lee $\mathrm{SH}$, et al. Differences in Temperament and Character Dimensions of Personality between Patients with Hwa-byung, an Anger Syndrome, and Patients with Major Depressive Disorder. Journal of Affective Disorders. 2011;138(1-2): 110-116.

20. Kim SY, Park JE, Seo HJ, Lee YJ, Jang BH, Son HJ, et al. NECA's Guidance for Undertaking Systematic Reviews and Meta-analyses for Intervention. Seoul:National Evidence-based Healthcare Collaborating Agency. 2011.

21. Kim MJ, Lee SY. Clinical Study on 29 Hospitalized Cases of Hwa-byung. Journal of Korean Medicine, Daejeon University. 1999;8(1): 783-792.

22. Kim JW, Hyun KC, Whang WW. A Study on the Origin of Hwa-byung. Journal of Oriental 
Neuropsychiatry. 1999;10(1):205-216.

23. Lee SH. A Study on the Hwa-byung (Anger Syndrome). Journal of Korea Hospital. 1977; 1(2):63-69.

24. Lee SG, Kim JW, Whang WW. A Case Study on Hwa-byung. Journal of Oriental Neuropsychiatry. 1996;7(1):173-180.

25. Association of Korean Medicine and Korea Institute of Oriental Medicine. Clinical Evidence of Korean Medicine. Seoul and Deajeon: Association of Korean Medicine and Korea Institute of Oriental Medicine. 2014:125-138.

26. Kim JW, Jung IC, Kang HW, Lee SG, Jung SW. Clinical Guidlines for Hwa-byung. Seoul: Korean Society Of Oriental Neuropsychiatry and Hwa-byung Research Center. 2013.

27. Bae DB, Lyu SJ, Lee GE, Lee SJ, Kang HW, Lyu YS. The Effects of Acupuncture Treatment on the Autonomic Nervous Systems of Hwa-byung Patients' Insomnia. Journal of Oriental Neuropsychiatry. 2014;25(3):235-242.

28. You SY, Kim MRH, Kim JH. Effects of Mindfulness Meditation on Symptoms of Depression, Anxiety, Stress, and Hwa-byung among Middle-aged Women with Hwa-byung Disorder. Korean Journal of Health Psychology. 2014;19(1):83-98.

29. Choi WC. The Effect of Sihogayonggolmoryeo -tang on Anxiety of Patients with Hwa-byung: Randomized, Double-blinded, Placebo-control Trial. Graduated School of Deajeon University. 2015.

30. Lee SG, Choi WJ, Kang HW, et al. Questionnaire Survey of Nonherbal Therapy of Hwa-byung in Professionals. Journal of Oriental Neuropsychiatry.
2013;20(2):133-141.

31. Min SK. Hwa-byung and the Psychology of Hahn. Journal of Korean Medicine. 1991;34(11): 1189-1198.

32. National Center for Complementary and Integrative Health. Mind and Body Interventions Information. Available from: URL: https://nccih. nih.gov/taxonomy/term/354.

33. Chung SY, Song SY, Kim JW. Clinical Guidelines for Hwa-byung II. (Research on the Status of Hwa-byung in Korea). Journal of Oriental Neuropsychiatry. 2013;24(1):15-22.

34. Textbook Compilation Committee of Neuropsychiatry of Korean Medical Schools in Nation, Neuropsychiatry of Korean Medicine, Jipmoondang, Seoul, Republic of Korea, 2011.

35. Textbook Compilation Committee of Meridian and Acupoint of Korean Medical Schools in Nation. Details of Meridians and Acupoints (Volume II); A Guidebook for College Students. Wonju:Yibang Publisher. 2009.

36. Textbook Compilation Committee of Meridian and Acupoint of Korean Medical Schools in Nation. Details of Meridians and Acupoints (Volume I); A Guidebook for College Students. Wonju:Yibang Publisher. 2009.

37. Kang HW, Lee SG, Lee JH, Park BR, Lyu YS. Clinical Guidelines for Hwa-byung IV. (Medications \& Acupuncture and Moxibustion). Journal of Oriental Neuropsychiatry. 2013;24(1): $37-46$.

38. Jung IC, Choi WC, Lee SR. Clinical Guidelines for Hwa-byung III. (Diagnosis and Assessment of Hwa-byung). Journal of Oriental Neuropsychiatry. 2013;24(1):23-36. 